\section{El Estado chileno, los lafkenche y la Ley 20.249: ¿Indigenismo o política del reconocimiento?}

The Chilean State, the lafkenche people and the Law 20.249: Indigenism or politics of recognition?

NicolÁs Gissi*

DANIELA IBACAChe*

BernaRdo Pardo ${ }^{* * *}$

Ma. Cristina ÑNCucheo ${ }^{* * * *}$
Departamento de Antropología, Universidad de Chile, Av. Capitán Ignacio Carrera Pinto $N^{\circ} 1045$, Ñuñoa, Santiago. Correo electrónico: ngissi@u.uchile.cl.

** Centro de Salud Familiar Aviador Acevedo, Aviador Acevedo 906, Quilpué, Región de Valparaíso. Correo electrónico: daniela. ibacache@gmail.com.

*** Escuela de Enfermería, Universidad Mayor, Sede Temuco, Avenida Alemania 0281, Temuco. Correo electrónico: bernardo.pardo@ uca.es.

**** Identidad Territorial Lafkenche, Carrera $\mathrm{N}^{\circ} 152$, Temuco. Correo electrónico: cnancucheo@gmail.com.

\section{Resumen}

Los mapuche-lafkenche iniciaron un movimiento social después de promulgada, en 1991, la Ley General de Pesca y Acuicultura (Ley 18.892), pues esta normativa no señalaba la presencia de los sujetos indígenas en las costas chilenas. Esta falta de reconocimiento trasladó las demandas indígenas desde las tierras del interior hacia el borde-mar, construyéndose hasta el día de hoy una breve historia (1995-2016) tanto de encuentros como de desencuentros entre los respectivos gobiernos de Chile y el pueblo mapuche-lafkenche. Después de más de una década de negociaciones, se promulgó en 2008 la Ley Lafkenche (Ley 20.249) que crea el espacio costero marino de los pueblos originarios (EMCPO) y reconoce a los pueblos indígenas su condición de habitantes del borde costero, del cual hacen distintos usos consuetudinarios (pesquero, religioso, recreativo). Sin embargo, en los años 2012 y 2013, los eventos que pudieron generar ciertas esperanzas de que este proceso de diálogo deviniera en el inicio de un renovado pacto entre chilenos y mapuche, hoy se ha transformado en una sensación de frustración, recordándonos las viejas políticas indigenistas.

Palabras clave: Mapuche-Lafkenche, Ley General de Pesca y Acuicultura, reconocimiento, borde costero, Ley Lafkenche.

\section{Abstract \\ The Mapuche-Lafkenche people started a social movement after promulgated, in 1991, the General Law of Fishing and Fish farming (Law 18.892), since this regulation did not indicating}


the presence of the indigenous peoples in the coasts of Chile. The lack of recognition produced a shift of the indigenous demands from the interior lands to the sea. This led to the construction of a brief ongoing story (1995-2016), which includes both encounters and misunderstandings between the respective governments of Chile and the Mapuche-Lafkenche people. After more than one decade of negotiations, the Lafkenche Law (Law 20.249, 2008) was created. It creates the coastal marine space of the original peoples (EMCPO) and recognizes the indigenous peoples' condition of inhabitants of the coast, as well as the traditional uses they give to this area (fishing, religious, recreational). Nevertheless, the events that could generate hope in the years 2012 and 2013 that this process of dialog would lead to the beginning of a renewed agreement between Chileans and Mapuche, have already transformed in a feeling of frustration, remembering us the old indigenists politicies.

Key words: Mapuche-Lafkenche, General Law of Fishing and Fish farming, recognition, coastal areas, Lafkenche Law.

\section{Introducción}

En este inicio de siglo, ya no resulta sorprendente para los chilenos la afirmación de que la solución al conflicto entre el Estado chileno y los pueblos originarios, en particular con el pueblo mapuche, trasciende un enfoque meramente económico. La idea de que se trataría solo de un problema de pobreza pierde año a año más fuerza. Las dimensiones económica, político-jurídica y cultural (simbólica) exigen ser reconocidas si se quiere comprender y comenzar a destrabar la fricción interétnica entre indígenas y chilenos no indígenas. Una u otra concepción del 'problema indígena' implicará una u otra política indígena. El no reconocimiento del carácter étnico del 'problema mapuche' conduce a desarrollar políticas enfocadas solo a superar la pobreza. Desde inicios del siglo XX, este tipo de políticas se han venido ejecutando, no solo en Chile sino que en toda América Latina. Se trata del indigenismo, que busca integrar la diversidad a una supuesta nación homogénea. En cambio, el reconocimiento del carácter étnico de la problemática conducirá a desarrollar políticas más complejas, multifactoriales, centradas esta vez en la participación y autogestión indígena a partir del principio de unidad en la diversidad.

El caso que aquí analizamos busca dilucidar y destacar cómo, después de un largo proceso de más de una década de diálogos y negociaciones entre los mapuche-lafkenche (mapuche del mar, ubicados entre el río Bío Bío y Chiloé) y los gobiernos de la época (19952008), se pasó de una inicial desconfianza a un fructífero intercambio de ideas, concluyendo con la creación de la denominada Ley 20.249 (Ley que crea el espacio costero marino de los pueblos originarios) el año 2008 y con el reconocimiento a los pueblos indígenas de su condición de habitantes del borde costero, del cual hacen distintos usos consuetudinarios (pesquero, religioso, recreativo).

Fue un proceso tenso para las partes, dada la presencia en ambos actores de activas memorias de larga duración sobre los difíciles vínculos chileno-mapuche. Durante los dos siglos republicanos, las disposiciones unilaterales, así como los intentos de diálogo y negociación, han sido múltiples. Algunos de ellos han dado lugar a acuerdos prontamente transgredidos, mientras en el último tiempo, a partir de una creciente sensibilidad internacional por las 
problemáticas indígenas y la recuperación de la democracia (post 1990), se han podido observar actos y decisiones gubernamentales orientadas a la legitimación de las demandas de los pueblos originarios, los que se han traducido, incluso, en cuerpos legales con pertinencia indígena. Debemos destacar al menos dos hitos fundamentales en este parcial acercamiento entre el Estado chileno y su diversidad étnica a partir de la última década del siglo XX: en primer lugar, la aprobación en 1993 de la Ley 19.253, conocida también como Ley Indígena -en la que el Estado reconoce la existencia de las etnias originarias que habitan en su territorio- $y$ 15 años después, la ratificación por el Estado chileno del Convenio N¹69 de la Organización Internacional del Trabajo (OIT). Se generan así esperanzas de que desde el Estado no solo lleguen las fuerzas policiales (police) sino que también se generen mejores políticas públicas (policies) referentes a los pueblos originarios. Al fin, se pensó, se estaba ante una política postindigenista, descolonizadora y democratizante.

En el año 2016, sin embargo, seguimos observando a través de los medios de comunicación un conflicto que se plasma en una escalada de violencia y muerte, frente a la cual el Estado chileno parece desintonizado, confundido respecto al tipo de política indígena a desarrollar, mientras el pueblo mapuchecontinúa movilizándose para lograr una salida que implica necesariamente el campo político, pues toda reivindicación pasa por el reconocimiento (Foerster y Vergara 2000; Bengoa 2000; Gissi 2002). Lo anterior en contraste con las políticas indigenistas, que sostienen que la integración social implica una creciente homogeneización cultural, tendiendo a la desaparición de las culturas indígenas y a la construcción de una nación mestiza (Stavenhagen 2007).
En su texto "Multiculturalismo y la política del reconocimiento", Taylor (1993) describe el problema del reconocimiento como propio de la modernidad y de las sociedades liberales. Previamente, el reconocimiento estaba ligado a una identidad basada en categorías sociales no cuestionables. Sin embargo, la modernidad trae consigo la necesidad de negociar el reconocimiento a partir de las interacciones:

\footnotetext{
[El reconocimiento] [d]ebe ganarse por medio del intercambio y el intento puede fracasar. Por ello ahora por primera vez se siente su necesidad. En la época premoderna, la gente no hablaba de 'identidad' y 'reconocimiento' no porque la gente no tuviera lo que denominamos identidades, o porque éstas no dependieran del reconocimiento, sino porque entonces eran demasiado poco problemáticas como para que fueran problematizadas como tales (Taylor 1993: 28).
}

El mismo autor describe dos miradas en torno a esta problemática en la modernidad. Por un lado, la interpretación universalista que establece el reconocimiento de los mismos derechos y obligaciones civiles para todos. $Y$ la posición opuesta, la política de la diferencia, que propone como objeto de reconocimiento la identidad particular y única de cada individuo y comunidad. Esta mirada del reconocimiento implica necesariamente que se ponga en juego, en la interacción, la forma en que cada comunidad representa e interpreta el mundo, su horizonte de sentido, constitutivo de su identidad. Es por ello que el reconocimiento implica un esfuerzo, muchas veces una confrontación, de dos formas de ver el mundo. El falso reconocimiento o la falta de reconocimiento pueden causar daño en las personas, grupos sociales y pueblos. Así, Taylor indica que ["d]entro de esta perspectiva, el falso reconocimiento no sólo muestra una falta del respeto debido. Puede infligir una herida dolorosa, que causa a sus víctimas un mutilador odio a sí mismas. El reconocimiento no sólo es 
una cortesía que debemos a los demás: es una necesidad humana vital" (Taylor 1993: 44-45).

Ahora bien, si esta falta de respeto a la dignidad del otro consiste no solo en desvaloración en la vida cotidiana sino que, además, se sostiene en una normativa legal, implicaría institucionalizar la consideración de que existen en Chile personas y colectivos carentes de un estatus adecuado para establecer una interacción igualitaria con los miembros no étnicos de la nación. Esta exclusión de derechos legales sería, por tanto, un hecho cultural, político-jurídico y económico, pues impediría a los pueblos indígenas el acceso a bienes que ancestralmente han usado. Se trataría, en primer lugar, de una falta de justicia distributiva, en la medida en que se les niega la posibilidad de ejercer libertades supuestamente garantizadas y legalmente instituidas para todos, así como recursos materiales. Pero también constituye una falta de justicia del reconocimiento (Honneth 1997), entendiendo que el origen de los principios de justicia está en las relaciones sociales. La justicia del reconocimiento propende, desde la institucionalidad, a asegurar las condiciones sociales para el reconocimiento mutuo en la interacción cotidiana.

Es en este contexto de lucha por el reconocimiento que -después de promulgada en 1991 la Ley General de Pesca y Acuicultura, que regula el uso de los recursos marítimos (Ley 18.892)- se inició un movimiento social mapuche-lafkenche, debido a que tal norma no reconocía la presencia indígena en las costas del país.

\section{Contexto jurídico nacional e internacional sobre pueblos indígenas}

La publicación por el Estado chileno de la ley №19.253 el 5 de octubre de 1993 (conocida como Ley Indígena), durante el gobierno del Presidente Aylwin, constituyó un primer paso en el camino al reconocimiento de los derechos de los pueblos indígenas. Más allá de los temas pendientes y las indicaciones que surgieron a partir de la aplicación de la Ley Indígena, esta norma reconoce la presencia de 'etnias' en el territorio nacional. El mundo indígena entendía que con esta ley se protegía su cultura y territorios. Dicha normativa, además, estableció la creación de la Corporación Nacional de Desarrollo Indígena (CONADI), pensada como un ente mediador entre el Estado y las etnias originarias ${ }^{1}$. Sin embargo, los estándares internacionales respecto al tema de derechos de pueblos indígenas son mucho más exigentes que los que esta ley señala, siendo el reconocimiento constitucional de los pueblos originarios -aunque de suma urgencia, según ha reconocido el segundo gobierno de la Presidenta Michelle Bachelet- un punto crucial y una deuda pendiente.

Los estándares internacionales tienen como base al Convenio N¹69 de la Organización Internacional del Trabajo (OIT), el que fue adoptado por la Conferencia General de la Organización Internacional del Trabajo en 1989 y que fue aprobado por el Congreso Nacional de Chile el año 2008, entró en vigencia en nuestro país el año 2009. En su Artículo $n^{\circ} 2$ indica:

La Ley 19.253 define que los indígenas de Chile son los descendientes de las agrupaciones humanas que existen en el territorio nacional desde tiempos precolombinos, que conservan manifestaciones étnicas y culturales propias siendo para ellos la tierra el fundamento principal de su existencia y cultura. En este artículo se utilizan los conceptos de pueblos indígenas y pueblos originarios como sinónimos. 
1. Los gobiernos deberán asumir la responsabilidad de desarrollar, con la participación de los pueblos interesados, una acción coordinada y sistemática con miras a proteger los derechos de esos pueblos y a garantizar el respeto de su integridad.

2. Esta acción deberá incluir medidas: a) que aseguren a los miembros de dichos pueblos gozar, en pie de igualdad, de los derechos y oportunidades que la legislación nacional otorga a los demás miembros de la población; b) que promuevan la plena efectividad de los derechos sociales, económicos y culturales de esos pueblos, respetando su identidad social y cultural, sus costumbres y tradiciones, y sus instituciones.

En el artículo 6 del Convenio N 169 de la OIT, se especifica que:

1. Al aplicar las disposiciones del presente Convenio, los gobiernos deberán: a) consultar a los pueblos interesados, mediante procedimientos apropiados y en particular a través de sus instituciones representativas, cada vez que se prevean medidas legislativas $o$ administrativas susceptibles de afectarles directamente;

2. Las consultas llevadas a cabo en aplicación de este Convenio deberán ejecutarse de buena fe y de una manera apropiada a las circunstancias, con la finalidad de llegar a un acuerdo o lograr el consentimiento acerca de las medidas propuestas.

Finalmente, para lo que aquí nos interesa destacar, en el artículo 13 del Convenio N¹69 de la OIT, se relacionan los conceptos de tierra y territorio:

Al aplicar las disposiciones de esta parte del Convenio, los gobiernos deberán respetar la importancia especial que para las culturas y valores espirituales de los pueblos interesados reviste su relación con las tierras o territorios, o con ambos, según los casos, que ocupan o utilizan de alguna otra manera, y en particular los aspectos colectivos de esa relación.

Casi dos décadas después, la Organización de las Naciones Unidas (ONU) logró un nuevo avance, a través de su Declaración de las Naciones Unidas sobre Derechos de los Pueblos Indígenas, la que fue aprobada el año 2007. La Declaración en sus fundamentos reconoce
El hecho de que los pueblos indígenas han sufrido injusticias históricas como resultado, entre otras cosas, de la colonización y de haber sido desposeídos de sus tierras, territorios y recursos, lo que les ha impedido ejercer, en particular, su derecho al desarrollo de conformidad con sus propias necesidades e intereses.

Los artículos 3, 25 y 26 sostienen explícitamente el derecho de los pueblos originarios a determinar libremente su desarrollo económico, social y cultural, así como el derecho a mantener y fortalecer su propia relación espiritual con las tierras, territorios, aguas, mares costeros y otros recursos que tradicionalmente han ocupado y utilizado.

En este sentido, la Ley Lafkenche se sitúa en este nuevo escenario jurídico, global y nacional, pero además se diferencia de cualquier normativa antes instaurada en el país, pues emerge desde las comunidades, a partir de las consecuencias de la Ley de Pesca y Acuicultura (1991). Tal como lo señalan las comunidades pertenecientes a la asociación Identidad Territorial Lafkenche en un comunicado público fechado el 21 de noviembre de 2007, una de las características centrales de esta ley ha sido que la propuesta surgió desde las propias organizaciones y en un proceso ampliamente participativo, mostrando cómo, tras un largo proceso de discusión y negociación, las comunidades fueron capaces de instalar -voluntad política mediante- sus preocupaciones y lograr que se legisle en torno a ellas.

Antes de referirnos explícitamente a este proceso participativo y de negociaciones entre el Estado chileno y el pueblo mapuche-lafkenche, respecto a la ley 20.249, intentaremos en el siguiente apartado hacer una síntesis de las principales características socio-culturales de los lafkenche, lo que nos permitirá constatar y 
comprender la existencia de una lógica distinta a la del Estado chileno.

\section{Cultura, autoridades y religiosidad lafkenche: breve caracterización}

Los lafkenche constituyen una de las seis identidades territoriales pertenecientes al pueblo mapuche (junto con pewenche, nagche, wenteche, puelche y williche), localizándose entre el río Bío-Bío y el Calle-Calle. El mar y las actividades económicas costeras tienen un fuerte sentido religioso en la cosmovisión mapuche-lafkenche, la que busca no perder los precarios equilibrios entre el cielo y la tierra, entre la tierra (lo sólido) y las aguas (lo líquido), entre las distintas manifestaciones naturales, así como entre los seres humanos y la naturaleza, y entre los propios seres humanos. Tales equilibrios deben propiciarse a través de variados ritos, destacando el nguillatun, ceremonia de oración propiciatoria del bienestar colectivo (Foerster, 1993; Bengoa, 2007).

Los ritos religiosos como el nguillatun son aún hoy encabezados o bien por el lonko o bien por el o la machi ("maestra de paz" en Chiloé) de la comunidad. El lonko es el articulador de la sociedad política mapuche. Cuando se presentan conflictos intra o intercomunidades, ellos deben mediar y buscar una solución. Interviene también cuando se realizan actividades sociales de importancia, como casamientos y ceremonias religiosas. El werken, por su parte, es un mensajero que ayuda al lonko en la interacción y negociaciones entre las distintas comunidades. Stuchlick (1974) señala que la autoridad del lonko es más consultiva que decisiva. Las discusiones y decisiones se realizan entre un grupo de hombres que representan la opinión pública, llegándose a acuerdos mutuos (en Hervé y Urrejola, 1994). Ahora bien, el no reconocimiento por parte de la sociedad chilena del papel mediador entre ésta/e y su comunidad, así como el carácter igualador de las leyes chilenas, han debilitado el poder del lonko. Progresivamente, se dejó de mencionarlo y se comenzó a hablar de "dirigente". Sin embargo, como parte de los procesos revitalizadores de la cultura/ cosmología/azmapu (derecho, en mapuzugun) mapuche de los últimos veinte años, su rol se ha vuelto a potenciar, aunque muchas veces compartiendo la representación comunitaria con un dirigente más joven, y más ligado a la sociedad e institucionalidad chilena.

El/la machi es la principal figura religiosa de las comunidadesmapuche-lafkenche. Losnguillatun lafkenche se han realizado históricamente en conexión con el mar, al cual se le entregan ofrendas y se le pide por la salud comunitaria. En distintos momentos del año, unas comunidades se invitan con otras, reforzando los lazos de reciprocidad. Usualmente el nguillatun se realiza entre dos o tres comunidades vecinas. Como señala Martínez (1995: 72):

El nguillatun permite la realización del azmapu, al tiempo que se constituía en un lugar privilegiado para llegar a acuerdos entre las comunidades y realizar el juego del palin [chueca]. Sociabilidad, realización ritual y acuerdos entre comunidades, fueron los elementos que, junto con el linaje, permitieron articular y reproducir la identidad territorial lafkenche, no disociado, sino que integrando la identidad particular con la identidad general del pueblo.

Tan importantes como los ritos, son para la religiosidad lafkenche, los mitos. Destacan el de trentren y kaykay, mito de origen mapuche, así como el de Sumpall y el abuelito Huenteao en el territorio lafkenche, todos los cuales hacen referencia a su vínculo con el mar. Sobre 
las aguas y su visión sagrada, señaló Bengoa recientemente, articulando lo material con la dimensión simbólica de la cultura (Martínez, 1995: 45):

En esas aguas viven aves, animales, peces, plantas y mariscos de todos los tamaños y formas y seres maravillosos [...] No tomar en serio esta dimensión espiritual del territorio significaría no comprender nada de la historia de la sociedad de los antiguos mapuche, nada del porqué de esta defensa impaciente de donde han vivido"

El autor hace especial referencia a Sumpall, un personaje que vive en el mar o un lago y que de tanto en tanto se lleva una mujer hacia las aguas, dejándolas encantadas. Un mito semejante en territorios lafkenche y williche es el del abuelito Huenteao, en el que se narra la historia de un hombre que llegó al mar desde el interior. Se recuerda que era un joven muy cercano a Dios/Ngenechen y que un día entró al mar y quedó encantado en roca. Desde ahí, en San Juan de la Costa, actúa como intermediario con Ngenechen, protegiendo a los mapuche, quienes inician sus rogativas o nguillatun junto a él (Foerster, 1985; Gissi, 1997). En Chiloé, por su parte, es reconocida la creencia en la Pincoya, una mujer joven y con un largo pelo rubio, que desde las profundidades del mar ayuda a los lafkenche.

Las normas de convivencia mapuche, entonces, así como de interacción con la naturaleza, el azmapu o admapu, provienen de los antepasados, y tuvieron un origen religioso. Son principios sagrados que se han transmitido de generación en generación, aceptándose tácitamente y transmitiéndose de forma oral (Hervé y Urrejola, 1994).

\section{Los lafkenche y la Ley Lafkenche}

Como vemos, los lafkenche se caracterizan como gente que define su lugar en el mundo a partir del vínculo con el mar. Sin esta relación se pierde el sentido histórico-espiritual de su identidad, como aquello que los distingue, por tanto:

\footnotetext{
Para éstos, existe la percepción de que el derecho al acceso y la utilización como elemento productivo del mar es un derecho que se tiene por el sólo hecho de ser lafkenche, sin necesidad de autorización, ni concesión por parte de autoridad, ni organismo alguno (BCN, 2008: 3).
}

La ley 20.249 reconoce a los indígenas su condición de habitantes del borde costero desde tiempos ancestrales. Es de destacar que esta normativa hace referencia a todos los pueblos originarios existentes actualmente en Chile, los que están reconocidos en la Ley $\mathrm{N}^{\circ}$ 19.253, sin embargo, como "etnias", esto es: aymaras, quechuas, atacameños, collas, diaguitas, rapanui, mapuche, kaweskar y yaganes. A partir de ello se crea la figura administrativa denominada Espacio Costero Marino de los Pueblos Originarios con el fin de resguardar el uso consuetudinario que han ejercido desde tiempos inmemoriales. Sin embargo, la ley se denomina usualmente como Ley Lafkenche dado el protagonismo que han alcanzado los mapuche-lafkenche con anterioridad (desde mediados de los años 90) y con posterioridad a la publicación de esta ley, debido a su manifiesto interés por contar con espacios costero-marinos que les permitan mantener sus prácticas económicas y usos culturales.

Si bien esta ley constituye un hito en la relación del Estado chileno con los pueblos indígenas y un avance en el proceso de reconocimiento y respeto por sus derechos, deja en evidencia una cuestión fundamental que cruza toda la 
política indígena. Se revela la existencia de dos lógicas culturales distintas: donde el Estado chileno "lee" territorio como un espacio/suelo destinado al emplazamiento, los mapuche -desde su cosmovisión/cosmología- entienden territorio como un concepto global que incluye la tierra, sus recursos y sus habitantes. Como señala Toledo (1996: 3):

Existe una contraposición entre el concepto indígena de Mapu que engloba todos los recursos -suelo, agua, riberas, subsuelo, bosques- y el concepto jurídico chileno que desvincula estos elementos en distintos regímenes de propiedad y concesión particulares.

Esta diferencia de enfoque entre ambos actores, Estado y comunidades lafkenche, es el origen de un conflicto instalado en el borde costero que refiere a la recuperación del lafkenmapu (litoral) por parte de los ocupantes ancestrales de este territorio. Esta "disputa territorial", que extiende los conflictos desde los espacios terrestres a los espacios costeros y marinos, no se da a partir de la promulgación de la Ley General de Pesca y Acuicultura durante los Gobiernos de la Concertación, sino que se remonta a la ocupación que el incipiente Estado de Chile realiza luego de la promulgación de la Constitución del año 1822 , en la zona sur del país. Continúa con las políticas desarrollistas de comienzos de los años 30 y logra su mayor exclusión desde el año 1988 durante el Régimen Militar, cuando se ingresa a discusión el proyecto de dicha norma. De esta forma la administración y el acceso a los recursos pesqueros es una de las tantas normas que buscan enajenar los recursos, en virtud de brindar certeza a los privados de la tenencia en propiedad o administrativa de los recursos naturales. Lo anterior se constata y perfecciona con la última modificación de la Ley de Pesca, según lo declarara el propio Ministro de Economía del gobierno de Sebastián Piñera (Longueira, 2012).

\section{Etapas del acuerdo chileno-mapuche: un desafío al porvenir}

Ahora bien, dilucidado el origen del problema, proponemos mostrar este proceso en tres momentos iniciales y un cuarto que nos permitirá visualizar la actual estrategia de la orgánica indígena para avanzar en la implementación de la Ley 20.249 .

\section{Etapa 1. Profundización del Conflicto}

Esta etapa puede distinguirse desde el año 1989 hasta comienzos del año 1995, y es un proceso que tiene su origen en la promulgación, por parte del Estado chileno, de dos cuerpos legales. A fines de la dictadura, el 20 de octubre del año 1988, se ingresa el Mensaje Presidencial que contiene el proyecto de la Ley General de Pesca y Acuicultura (Ley $\left.N^{\circ} 18.892 / 1989\right)$. Esta ley establece el marco normativo del sector pesquero nacional y tiene por objeto generar un régimen jurídico que indique reglas para este sector, resguardando en forma eficiente la preservación de los recursos hidrobiológicos, y que permita la existencia de un sistema permanente donde se desenvuelvan armónicamente, derechos y deberes, tanto del Estado como del sector privado (BCN, 1989).

Desde el ingreso de este proyecto, las comunidades lafkenche, así como los williche (gente del sur) y pewenche (gente del piñón), mostraron su preocupación por lo que definieron como "una nueva imposición legislativa que se agrega a derechos que anteriormente también han sido violados". Este nuevo marco jurídico es visto como una amenaza ya que permitía, en un primer momento, seguir entregando la concesión de playas e islas mapuche-lafkenche a empresas nacionales y transnacionales, lo que pone en peligro la flora y fauna de la zona 
costera y amenaza la integridad de la propiedad indígena. En un segundo nivel propiciaba el despojo de las tierras, riberas y recursos marinos en zonas puntuales del borde costero, las que, por derechos ancestrales, las comunidades perciben como propias y que podría llevarse a cabo mediante la declaración de Parque Marino de la zona o la concesión de licencias de explotación privada de los recursos marinos.

El proyecto de ley significaba una amenaza para las comunidades pues establecía una medida administrativa sobre los recursos bentónicos, denominadas Áreas de Manejo y Explotación de Recursos Bentónicos (AMERB). La puesta en marcha de esta medida mediante la promulgación del Decreto Supremo $\mathrm{N}^{\circ}$ 355/1995 (Ministerio de Economía, Fomento y Reconstrucción) ha implicado la pérdida sustancial de espacios de interés comunitario, desde Arauco hasta Chiloé. Solamente en la Región de Los Lagos se han decretado cerca de 296 AMERB, las que han sido entregadas en su mayoría a organizaciones de pescadores artesanales (130 de 137) (SERNAPESCA, 2013).

Por otro lado, la Ley Indígena/1993, dejó en un segundo plano las demandas que no están vinculadas con la tenencia de tierras, asociadas en la mayoría de los casos a propiedades que hoy están bajo títulos privados y no enfrenta lo que ocurre con los bienes nacionales de uso público, como son las playas, terrenos de playas y en general el espacio definido como Borde Costero por el Estado chileno².

Comprende los terrenos de playas fiscales situados en el litoral, la playa, las bahías, golfos, estrechos y canales interiores y el mar territorial de la República, encontrándose sujetos al control, fiscalización y supervigilancia del Ministerio de Defensa Nacional, Subsecretaría de Marina (Decreto Supremo № 475, MINDEF, SUBSECMAR, Política Nacional de Uso del Borde Costero del Litoral de la República, de Enero de 1995).
A este respecto es importante destacar que en el Primer Informe Comisión Especial Pueblos Indígenas, presentado a la Cámara de Diputados, en el proceso de discusión de la Ley Indígena (BCN, 1993), se menciona al pueblo mapuche con sus diferentes regiones, hoy conocidas como identidades territoriales, entre las que se distinguen los lafkenche (lafkenmapu) y williche (willimapu), sin embargo dicha distinción no es rescatada en la Ley que finalmente fue promulgada, aunque el proyecto sí hace mención a los temas de borde costero y sus recursos.

Lo indicado anteriormente permite constatar que en el mundo político estatal las comunidades lafkenche no son consideradas actores vinculados a la pesca, ni al borde costero, poniendo en riesgo su sustentabilidad. $Y$ si bien los williche son relevados en los Artículos 60 y 61 de la Ley Indígena, éstos se refieren al sistema tradicional de cacicazgos y su ámbito territorial y sobre cómo el Estado se vinculará con ellos, no haciéndose mención explícita a su relación con el borde costero y sus recursos.

\section{Etapa 2. Negociación con el Estado}

Las situaciones planteadas anteriormente alertaron a un grupo de dirigentes de las comunas de Tirúa y Carahue, quienes entre los años 1995 y 2008 instalaron un proceso de negociación con el Estado, con la finalidad de resguardar el lafkenmapu. En paralelo y como estrategia que permitiera evitar la pérdida de espacios, muchas comunidades deciden solicitar las AMERB, mediante organizaciones funcionales, como sindicatos de pescadores artesanales y asociaciones indígenas, y no utilizando la orgánica mapuche (Identidad Territorial Lafkenche, 2004a). Durante el 
gobierno de Eduardo Frei Ruiz Tagle (19942000), se concretan una serie de reuniones con el Subsecretario del Ministerio de Planificación (MIDEPLAN), y con el de Pesca (SUBPESCA), así como con diversos legisladores para tratar los problemas que la aplicación de la normativa pesquera ocasionó a los mapuche-lafkenche y buscar una solución a éstos.

A partir del año 2000 e iniciado el gobierno de Ricardo Lagos (2000-2006), las organizaciones lafkenche profundizan su trabajo $\mathrm{y}$, como movimiento, comienzan un proceso de reestructuración territorial, logrando articularse con distintos espacios territoriales extendidos desde Arauco, por el norte, hasta Valdivia por el sur, abarcando las Interlocalidades de Huentelolén, Contulmo, Lleu Lleu, Pu Lafkenche, Newen Pu Lafkenche, Lof Budi, Porma-Malalwe y Maikillawe Koyawe Mapu. Esta articulación de comunidades adopta el nombre de Identidad Territorial Lafkenche.

A fines del año 2002, en el Palacio de La Moneda, el Presidente Lagos "reconoce la omisión y la amenaza que la ley de pesca significaba al no reconocer el legítimo derecho al mar de los lafkenche". Se acuerda entonces elaborar una propuesta por parte de la organización mapuche, pertinente a la propia cultura/ cosmología, que sirviera como base para la política pública, propiciando la búsqueda de una solución definitiva al problema (Identidad Territorial Lafkenche, 2004b).

La propuesta en cuestión sería entregada al Presidente Lagos el día 10 de Marzo de 2004 en un gran encuentro en el sector de Weicha, Trawa-trawa, territorio Budi, Región de La Araucanía, evento al que el Presidente no llegó (Millahual, 2004). Un par de meses después fue el mismo gobierno el que retomó las conversaciones con la dirigencia y concretó una reunión en la Moneda, donde finalmente se entrega la propuesta elaborada y se le plantean las problemáticas allí reseñadas.

El proceso que se inicia a partir de ese momento implica un esfuerzo de ambas partes, las que resuelven establecer una mesa de trabajo con objeto de redactar una indicación a la ley de pesca que disminuyera las externalidades negativas y los conflictos generados en su implementación. La estrategia de trabajo reveló posturas contrapuestas, reflejo de dos lógicas culturales no poco distantes: a) el gobierno, que propone la adopción de medidas temporales y la posibilidad del establecimiento de subsidios, inclinándose por una solución que aborde el aspecto económico, dejando en segundo plano lo organizacional y el control territorial, y b) la dirigencialafkenche, que entiende que la solución debe darse a partir de un proceso participativo de las comunidades, por lo que es de vital importancia que puedan reunirse internamente para poder expresar sus opiniones y llegar a consensos que se plasmen en ese documento. Cultura y política se cruzaban una vez más en los diálogos entre mapuche y chilenos. Indigenismo o política del reconocimiento volvía a ser el gran impasse.

De esta forma, el 13 de enero del año 2005 se establece un primer encuentro en el cual el gobierno es representado por los Subsecretarios de Pesca y MIDEPLAN, mientras la Identidad Lafkenche se hace presente por medio de su directiva y un dirigente delegado de cada territorio. El primer acuerdo de esta reunión es que ambas partes (gobierno y organización mapuche) trabajen de manera conjunta, a partir de lo cual se establece como prioritario que 
cada una de ellas cuente con un equipo técnico y político que pueda ir precisando conceptos y se reúna con su contraparte de manera periódica, a fin de ir concretando la indicación mencionada.

Visto que los puntos de partida que tienen ambos equipos de trabajo resultan disímiles, y entendiendo las particularidades que se requiere asumir para solucionar la demanda indígena, la contraparte de gobierno señala que dadas las características de la propuesta, no es posible elaborar una indicación a la ley de pesca. Lo que se requiere es una nueva institucionalidad que debe crearse a partir de una ley distinta. A partir de este momento el gobierno se compromete a ingresar un proyecto de ley que genere una institucionalidad ad hoc, que represente el sentido de lo demandado y expuesto por la Identidad Territorial Lafkenche y que permita ampliar, desde el prisma jurídico-administrativo occidental, el acceso y control del lafkenmapu. Luego de que cada uno de los equipos indicara los alcances de las discusiones, la generación del proyecto de Ley tendría una duración cercana a los ocho meses, en los cuales ambos equipos de trabajo establecerían acuerdos y discordancias. Los dirigentes mapuche eran conscientes de que se estaba haciendo historia, o al menos, que lo intentarían: no se trataba sólo de una indicación, de un ajuste a lo ya existente; el objetivo era una nueva institucionalidad.

\section{La metodología de la Identidad Territorial} Lafkenche implicó:

1. Sistematizar acuerdos y discordancias, y discutir éstas de manera permanente con una asamblea abierta, que contara con la representación de todos los territorios.

2. Estos acuerdos eran presentados a las bases por los dirigentes que asistían a la asamblea. En el caso de los puntos discordantes, éstos igualmente eran expuestos y discutidos, considerando en todo momento criterios de carácter cultural en la definición de "pisos de negociación".

3. El resultado de estas conversaciones locales era llevado a la asamblea, para que luego el equipo de la Identidad Territorial Lafkenche los presentara a su contraparte del gobierno.

Finalmente, se generó el proyecto de Ley que crea el Espacio Costero Marino de Pueblos Originarios, a partir de un proceso en donde ambos equipos fueron aportando y cediendo frente a argumentos de uno o de otro lado. El 24 de agosto de 2005, a través del mensaje $\mathrm{N}^{\circ}$ 127-353, la Presidenta de la República Michelle Bachelet da inicio al trámite del Proyecto de Ley que crea el Espacio Costero Marino de Pueblos Originarios. El proyecto ingresado está compuesto por 16 artículos distribuidos en seis títulos.

Desde el ingreso del Mensaje Presidencial al poder legislativo, el día24 deagosto del año2005, hasta el 16 de febrero de 2008, fecha en que se publica en el Diario Oficial para ser establecida como Ley de la República, transcurren 2 años y medio ${ }^{3}$. Durante todo este proceso la dirigencia y el equipo técnico mantienen un rol estratégico en las discusiones y sensibilización de las y los parlamentarios, centrado en la necesidad de aprobar una Ley que se ajuste lo más posible a lo presentado en el proyecto inicial. En esta línea, la ley originada por este proyecto es una contribución importante a la prevención y

Durante los dos años y cinco meses que dura la discusión del proyecto de ley, pasa por cuatro trámites constitucionales $(\mathrm{BCN}$, 2008):

- Primer Trámite Constitucional: Cámara de Diputados, 24 de agosto de 2005. Comisión de Recursos Naturales, Bienes Nacionales y Medio Ambiente.

- Segundo Trámite Constitucional: Senado, 16 de mayo de 2007. Comisión de Medio Ambiente y Bienes Nacionales.

- Tercer Trámite Constitucional: Cámara de Diputados. 20 de noviembre de 2007.

- Cuarto Trámite: Tribunal Constitucional, 04 de diciembre de 2007. 
solución de situaciones de conflicto respecto del uso de los recursos litorales, que busca así evitar lo que actualmente sucede con la tierra.

\section{Etapa 3. Implementación de la Ley 20.249}

Luego de la promulgación de la Ley 20.249 se esperaba un número importante de solicitudes, al menos una por cada organización de hecho o de derecho que participó en el proceso de discusión de la Ley. Unas 20 distribuidas entre la Provincia de Arauco y Aysén. Sin embargo, hasta Abril de 2015 sólo cinco de los territorios que originalmente participaron de las negociaciones -Lof (comunidad) Huentelolen, Lleulleu, Budi, Porma-Malalwe y Maikillawe Koyawe Mapuhabían realizado presentaciones, según lo indica la página institucional de SUBPESCA.

Detenerse a analizar por qué sólo algunas de las organizaciones que participaron del proceso ha realizado solicitudes, resulta interesante. Una hipótesis que se puede analizar es la probabilidad de éxito que tienen las solicitudes una vez que ingresan su postulación. Se deben distinguir aquí los puntos críticos de la postulación a un ECMPO: a) definir la organización que solicitará el ECMPO (comunidad o asociación de comunidades); b) el análisis de uso consuetudinario que realiza la CONADI; c) el pronunciamiento que realiza la Comisión Regional de Uso del Borde Costero (CRUBC). Este último punto ha resultado un obstáculo casi insalvable: la CRUBC ha limitado la asignación de solicitudes, ya que en virtud de las facultades que le otorga la Ley (Art. 8), con excepción de cuatro casos cuyas superficies son muy reducidas, el resto ha sido aprobada con modificaciones (las solicitudes de la Región de La Araucanía) o rechazadas (las de Los Lagos). Lo anterior podría explicarse debido a que la propuesta de zonificación del Borde Costero de la Región de La Araucanía reconoce una Zona de Desarrollo Indígena (ZDI), situación que no ocurre en el caso de la Región de Los Lagos, donde además el Ejecutivo ha obrado arbitrariamente según lo fallado por la Corte Suprema (causa rol 7544-2012).

Tras siete años (hasta Abril 2015) de promulgada la Ley Lafkenche, sólo se han decretado tres ECMPO y ocho están en proceso de destinación. En parte importante, esto se debe a que la ley no señala a la CRUBC criterios de decisión, de tal manera que goza de amplias facultades para emitir su pronunciamiento. Hoy día es indudable que el número de solicitudes se ha incrementado, llegando a 35 en total. Sin embargo, existen nudos críticos que aún no se han podido resolver, existiendo cinco solicitudes a las cuales las comunidades han renunciado, ubicándose en territorios emblemáticos: San José de la Mariquina, Valdivia, Toltén y San Juan de la Costa.

\section{Etapa 4. Estrategias de continuidad}

Los obstáculos en el proceso de implementación de la Ley Lafkenche ha implicado un cambio en la estrategia asumida por los dirigentes de la Identidad Territorial Lafkenche respecto a la elaboración de las próximas solicitudes y sobre las negociaciones con el gobierno. Pareciera que la dirigencia ha decidido enfrentar el proceso de recuperación territorial desde una perspectiva distinta, lo que se refleja en la activa participación que tuvieron en la discusión de la Ley 20.657 (BCN, 2013), que modifica la Ley 18.892 (Ley General de Pesca y Acuicultura). Este cambio normativo tiene por objeto:

a) Garantizar el uso sustentable de los recursos pesqueros.

b) Reformar el proceso de toma de decisiones. 
c) Proponer un cambio a la regulación del acceso a los recursos hidrobiológicos.

d) Modificar la regulación al sector pesquero artesanal.

e) Proponer el fraccionamiento de las principales pesquerías compartidas entre los sectores pesqueros artesanal e industrial, a partir de un acuerdo intersectorial.

Los puntos anteriores fueron interpretados por la dirigencia como una nueva amenaza, frente a la pérdida de derechos de acceso y asignación de cuotas, ya que deja fuera a los pueblos originarios, que milenariamente se han desarrollado junto al mar (Millabur, 2012). La discusión normativa por parte del Ejecutivo se funda en que la Ley 20.657 no afecta ni pone en riesgo los intereses de las comunidades, ya que éstas están protegidas por la ley 20.249. Sin embargo, dicha ley establece que esta norma se encuentra relacionada con la afectación del borde costero y en ningún momento resguarda el acceso equitativo a la administración de los recursos bio-pesqueros.

Ambas tesis se enfrentaron en la presentación de inconstitucionalidad que un grupo de senadores ${ }^{4}$ hicieron en el Tribunal Constitucional -bajo los roles 2387-12 y 2386-12- el año 2012, presentación que se basa en los siguientes elementos:

a) El proyecto vulnera la igualdad ante la Ley ya que propone enfoques distintivos para las comunidades rapa-nui, no así para los lafkenche, comunidades de Chiloé y de los canales australes.

b) Debió haber un diálogo de buena fe y con la intención de lograr un acuerdo con ellos, en el marco del deber de consulta de los artículos $6^{\circ}$ y $7^{\circ}$ del Convenio 169 de la OIT, ratificado por Chile y vigente, normas que son autoejecutables de acuerdo a lo resuelto por la Magistratura en sede de control preventivo del Convenio 169 de la OIT.

Senadores Eugenio Tuma, Ricardo Lagos Weber, Jaime Quintana, Guido Girardi, Ximena Rincón, Mariano Ruiz-Esquide, José Antonio Gómez, Isabel Allende, Pedro Muñoz, Alejandro Navarro y Camilo Escalona.
Es de considerar también que el Estado debe fijar cuotas de extracción sustentable, en el contexto del principio de preservar la naturaleza, contemplado en el numeral $8^{\circ}$ del artículo 19 de la Carta Fundamental. De esta forma la última modificación de la Ley de Pesca le asigna a unos pocos, por tiempo indefinido y mediante licencias gratuitas a 20 años renovables, los peces presentes y futuros, privando al resto de los chilenos, indígenas incluidos, de esta actividad económica invocando supuestos derechos históricos de los grupos económicos (STC, 2013).

Así, se sostiene la inconstitucionalidad de las basesdel proyectodeley, quepretendejustificarse teniendo como objetivo la sustentabilidad de los recursos hidrobiológicos, cuando en realidad resulta en la sustentabilidad financiera de la gran pesca. Agregan también como vulnerado el artículo $19, \mathrm{~N}^{\circ} 23$, constitucional, en cuanto al derecho a desarrollar una actividad económica lícita, e invocan el artículo 15.1 del Convenio 169 de la OIT, relativo a la protección de los recursos naturales en territorios indígenas, para después sostener la nulidad de derecho público del proyecto, por omisión de la consulta y alegar como vulnerada la garantía del contenido esencial de los derechos, del numeral $26^{\circ}$ del artículo 19 de la Carta Fundamental (STC, 2013)

El 23 de enero del 2013, el Tribunal Constitucional emite un pronunciamiento desfavorable a las comunidades, indicando "Desestimar las inconstitucionalidades alegadas en los requerimientos roles $\mathrm{N}^{\circ} 2387-12$ y 2388-12" (STC, 2013), lo que indudablemente establece un nuevo desafío respecto a lograr una recuperación de los recursos bio-pesqueros, evitando que, de esta forma, se repliquen los conflictos que hoy se dan respecto a las tierras 
del interior, como asimismo, continuar con antiguas políticas indigenistas.

\section{Palabras de Cierre}

A lo largo de las páginas anteriores planteamos que en el contexto de la "lucha por el reconocimiento" entre los pueblos originarios y el Estado chileno, los mapuche-lafkenche iniciaron un movimiento social después de promulgada, en 1991, la Ley General de Pesca y Acuicultura (Ley 18. 892), normativa que no señalaba la presencia de los sujetos indígenas en las costas del país. Este hecho de falta de reconocimiento, de valoración social y derechos legales, trasladó las demandas indígenas desde las tierras del interior hacia el borde-mar, construyéndose hasta el día de hoy una breve historia (1995-2015), tanto de encuentros como de desencuentros.

Como vemos, "el conflicto indígena no sólo se presenta en la tierra", según señala una noticia aparecida en el diario El Mercurio del mes de enero del año 2013. La Ley de Pesca se refiere a los pescadores artesanales e industriales, pero no considera a las comunidades indígenas que han usado ancestralmente el territorio costero. No se realizó tampoco la consulta respectiva, como indica el Convenio 169 de la OIT, violentándose los derechos indígenas.

La propuesta de la Ley Lafkenche surgió a partir de un proceso ampliamente participativo, logrando las comunidades y organizaciones que se consideraran sus preocupaciones y expectativas. Sin embargo, las tensiones, culturales y políticas, entre ambos actores, Estado y pueblo mapuche-lafkenche, no han dejado de estar presentes, lo que se evidencia en la casi inexistencia -después de seis añosde solicitudes de EMCPO aceptadas.

A partir de esta ley y después de haber acreditado la CONADI los usos consuetudinarios señalados en su solicitud por las comunidades de Punta Capitanes, en la comuna de Fresia, el Ministerio de Economía le entregó 104 hectáreas de borde costero a los lafkenche, convirtiéndose Punta Capitanes en la primera área que el Estado chileno entrega a comunidades indígenas para uso pesquero y/o religioso y/o recreativo. Están pendientes más de 30 solicitudes, las que suman más de 700.000 hectáreas de mar y borde-mar.

La Ley 20.249 es la primera norma que logra sentar en la misma mesa al Estado chileno y a las comunidades indígenas -en este caso lafkenche- y generar una salida jurídica a una demanda histórica. Este proceso representa una oportunidad que puede gatillar procesos similares, demostrando en los hechos la superación de políticas indigenistas, no reconocedoras de la diferencia étnica, características del siglo XX. Sin embargo, la forma con que actuó el gobierno de la Alianza por Chile, según lo indica la propia Corte Suprema, pone en riesgo este tipo de aprendizajes y valida otros procesos reivindicativos. Vemos así que esta normativa hace evidente una cuestión que cruza toda la política indígena: la existencia de dos posturas contrapuestas, que responden a una lógica y a una visión de mundo distinta. Mientras el Estado concibe "tierra" como un espacio destinado al emplazamiento, los mapuche la entienden como territorio, un espacio global que incluye el medio ambiente, sus recursos naturales y habitantes (SCS, 2012).

De este modo, una ley e institucionalidad que prometían ser un caso exitoso de política de 
reconocimiento, marcando el inicio de acciones orientadas hacia un encuentro intercultural, quedó una vez más trunco, confirmando a quienes sostienen que estamos ante dos cosmologías diferentes y opuestas, y que resulta poco viable una articulación de la cosmológica mapuche al interior del Estado-nación chileno. Surge así, nuevamente, la duda respecto a que ambas subjetividades puedan convivir, pacíficamente.

No es menor la relevancia que tiene el futuro de estos diálogos y negociaciones entre los mapuche costeros y el Estado chileno. Dado que el Estado es visto por parte del pueblo mapuche como campo de negociación y de deudas, resultando inseparable de la sangrienta "Pacificación" (1869-1883) y del anhelado reconocimiento, un buen proceso dialógico entre las asociaciones lafkenche y el Estado chileno mucho podría contribuir en el desafío de lograr un nuevo pacto nacional mapuchechileno, evitando que continúe la "espiral de violencia" en la Araucanía, en el que la muerte del matrimonio Luchsinger-Mackay y Matías Catrileo son quizás dos de los hechos más recordados. Todos ellos permanecen presentes en la memoria colectiva mapuche y chilena. Sin embargo, los eventos que pudieron generarnos ciertas esperanzas en los años 2012 y 2013 en que este proceso de diálogo deviniera en el inicio de un renovado acuerdo entre chilenos y mapuche, hoy ya se ha transformado en una sensación de frustración, recordándonos las antiguas políticas indigenistas.

Aun así podemos señalar que, aunque no constituye una política de reconocimiento propiamente tal, la promulgación de la Ley Lafkenche es un precedente de voluntad política de diálogo intercultural, de la posibilidad de establecer un proceso participativo con las comunidades y de abrir un espacio de validación de las inquietudes, demandas y propuestas de todas las partes. Es cierto que la aplicación de la normativa nos ha mostrado que el Estado chileno sigue teñido de un profundo indigenismo, pero no es menos cierto que es responsabilidad de todos, chilenos e indígenas, relevar lo ocurrido con la Ley Lafkenche como un primer e importante acercamiento. Una verdadera política de reconocimiento en relación a los pueblos originarios sólo será posible a partir de un cambio cultural y sabemos que estos cambios, aunque de largo aliento, comienzan con pequeñas -pero significativas- acciones.

\section{Bibliografía}

Bengoa, José. 2000. La emergencia indígena en América Latina, Santiago: FCE.

Bengoa, José. 2007. Historia de los antiguos mapuche del sur. Desde antes de la llegada de los españoles hasta las paces de Quilín. Siglos XVI y XVII, Santiago: Catalonia.

Biblioteca del Congreso Nacional. 1989. Historia de la Ley $N^{\circ}$ 18.892 Ley general de Pesca y Acuicultura. Pp. 1688. Disponible en: http://www.bcn.cl (consultado en marzo de 2014).

Biblioteca del Congreso Nacional. 1993. Historia de la Ley $N^{\circ}$
19.253 Establece normas sobre protección fomento y desarrollo de los indígenas y crea la Corporación Nacional de Desarrollo Indígena. Pp. 701. Disponible en: http://www.ben.cl (consultado en agosto de 2013).

Biblioteca del Congreso Nacional. 2008. Historia de la Ley $\mathrm{N}^{\circ}$ 20.249 Crea el espacio Costero Marino de los Pueblos Originarios. Disponible en: http://www.bcn.cl (consultado en agosto de 2013).

Biblioteca del Congreso Nacional. 2013. Historia de la Ley $N^{\circ}$ 20.657 Modifica en el ámbito de la sustentabilidad de recursos 
hidrobiológicos, acceso a la actividad pesquera industrial y artesanal y regulaciones para la investigación y fiscalización, la Ley General de Pesca y Acuicultura contenida en la Ley Nº 18.892 y sus modificaciones. Pp. 3631. Disponible en: http://www.bcn.cl (consultado en abril de 2013).

El Mercurio 06-01-2013. "Gobierno inicia entrega de áreas de mar a mapuche de la costa”, Santiago.

Foerster, Rolf y Vergara, Jorge. 2003. "Etnia y nación en la lucha por el reconocimiento. Los mapuche en la sociedad chilena", en H. Gundermann et al., Mapuche y aymaras. El debate en torno al reconocimiento y los derechos ciudadanos, Universidad de Chile, PREDES, Santiago: Ril.

Foerster, Rolf. 1985. Vida religiosa de los huilliches de San Juan de la Costa, Santiago: Rehue.

Foerster, Rolf. 1993. Introducción a la religiosidad mapuche, Santiago: Universitaria.

Gissi, Nicolás. 1997. Aproximación al conocimiento de la memoria mapuche-huilliche en San Juan de la Costa, Tesis para optar al título de antropólogo social, Facultad de Ciencias Sociales, Santiago: Universidad de Chile.

Gissi, Nicolás. 2002. "Los mapuche en el Santiago del siglo XXI: desde la ciudadanía política a la demanda por el reconocimiento", en revista Werken N³, 5-19. Disponible en: http:// www.revistawerken.cl/lectura/planilla2. php?ed=03\&art=001 (consultado en junio de 2014).

Gissi, Nicolás; Pardo, Bernardo; Ñancucheo, Cristina. 2013. Espacio costero marino de pueblos originarios (ECMPO): construcción participativa de una nueva institucionalidad de borde costero indígena. Manuscrito.

Grupo de las Naciones Unidas para el desarrollo. 2008. Directrices sobre los asuntos de los pueblos indígenas. Disponible en: http://www2.ohchr.org/english/issues/indigenous/docs/UNDGDirectrices_pueblos_indigenas.pdf (consultado en julio de 2013).

Hervé, Dominique; Urrejola, Antonia. 1994. El derecho consuetudinario indígena: su reconocimiento por el derecho internacional, el derecho comparado y el derecho chileno. El caso mapuche, Memoria de prueba, Facultad de Derecho, Santiago: Universidad de Chile.

Honneth, Axel. 1997. La lucha por el reconocimiento. Por una gramática moral de los conflictos sociales, Barcelona: Grijalbo.

Huilcaman, Aucán. 2013. Entrevista: "No estamos haciendo una reivindicación, sino apelando a tratados ya existentes". Disponible en: http://www.mapuexpress.net//index.php?act=news\&id=9727 (consultado en marzo de 2013).

Identidad Territorial Lafkenche. 1999. De la deuda histórica nacional al reconocimiento de nuestros derechos territoriales. Documento interno.

Identidad Territorial Lafkenche. 2004a. "Kiñeupe Taiñ Rakizuam Nietuam Kume Az Moñgen Taiñ Füxa Lafken Mapu-Mew. Unamos nuestro pensamiento mapuche para ejercer nuestro derecho al lafkenmapu". Propuesta Lafkenche. Documento interno.

Identidad Territorial Lafkenche. 2004b. "Carta abierta al Presidente Ricardo Lagos. Cronología de un diálogo frustrado".
Declaración Pública. Disponible en: http://www.mapuche-nation. org/espanol/html/noticias/carta-30.htm. (consultado en marzo de 2013).

Identidad Territorial Lafkenche. 2007. "Carta abierta de las comunidades mapuche lafkenche de la Identidad Territorial Lafkenche a la opinión pública". Disponible en: http://www. identidadlafkenche.cl/declaraciones/decl12.html. (consultado en marzo de 2013).

Ley N¹8.892. 1991. Ley General de Pesca y Acuicultura, Santiago.

Ley $N^{0} 19.253 .1993$. Establece normas sobre protección, fomento y desarrollo de los indígenas, CONADI, Temuco.

Ley No20.249. 2008. Ley Lafkenche, que crea el espacio costero marino de los pueblos originarios, Santiago.

Libertad y Desarrollo. 2010. "Conflicto mapuche: lecciones de la huelga de hambre". Disponible en: http://www.lyd.com (consultado en marzo de 2013).

Longueira, Pablo. 2012. Entrevista: Chile gana con la aprobación de la Nueva Ley de Pesca y Acuicultura. Disponible en: $\quad$ http://www.economia.gob.cl/2012/12/19/chile-gana-conla-aprobacion-de-la-nueva-ley-de-pesca-y-acuicultura.htm. (consultado en abril de 2013).

Martínez, Cristián. 1995. Comunidades y territorios Lafkenche. Los mapuche de Rucacura al Moncul, Temuco: IEI, UFRO.

Millabur, Adolfo. 2012. Entrevista: Los lafkenche y la ley de Pesca: ¿Por qué marchamos? Disponible en: http://www. theclinic.cl/2012/12/04/los-lafkenche-y-la-ley-de-pesca-por-quemarchamos/ (consultado en marzo de 2013).

Millahual, Carlos. 2004. "El desaire de Ricardo Lagos". En Periódico Mapuche Azkintuwe. Año $1 \mathrm{~N}^{\circ} 5$. Disponible en: http://www.archivochile.com/Pueblos_originarios/Info_prensa/ azkintuwe_05.pdf. (consultado en marzo de 2013).

Naciones Unidas. 2007. Declaración de las Naciones Unidas sobre los derechos de los pueblos indígenas. Disponible en: http://www.un.org/esa/socdev/unpfii/documents/DRIPS_es.pdf (consultado en marzo de 2013).

Organización Internacional del Trabajo (OIT). 1989. Convenio 169 sobre pueblos indígenas y tribales en países independientes. Disponible en: http://www.politicaspublicas.net/panel/ convenio169oit.html (consultado en marzo de 2013).

Salas, Ricardo. 2013. "Desde el Reconocimiento a la Interculturalidad", en Cuadernos del Pensamiento Latinoamericano N²0, Disponible en: http://www.cuadernoscepla.cl/web/wpcontent/uploads/Texto-4 Ricardo-Salas.pdf

Sentencia Corte Suprema. 2012. ROL 7544-2012. Disponible en: http://bibliotecadigital.indh.cl/bitstream/ handle/123456789/601/8b. - \%20Sentencia\%20C. \%20 S.? sequence=16 (consultado en agosto 2016).

Sentencia Tribunal Constitucional. 2013. ROL 2387-12. Disponible en: http://webcache.googleusercontent.com/ search?q=cache:jrqeQz8KviAJ:www.tribunalconstitucional.cl/ wp/descargar_expediente.php\%3Fid\%3D44905+\&cd=2\&hl=es$419 \& c t=c l n k \& g l=c l$ (consultado en agosto 2016). 
Sentencia Tribunal Constitucional. 2013. ROL 2388-12. Disponible en: http://webcache.googleusercontent.com/ search?q=cache:jrqeQz8KviAJ:www.tribunalconstitucional.cl/ wp/descargar_expediente.php\%3Fid\%3D44905+\&cd=1\&hl=es$419 \& \mathrm{ct}=\mathrm{clnk \& gl}=\mathrm{cl}$ (consultado en agosto 2016).

Servicio Nacional de Pesca (SERNAPESCA). 2013. GTI Área de Manejo. Base de datos. Disponible en http://www.sernapesca. $\mathrm{cl} /$ index.php?option=com_remository\&ltemid=246\&func=startdo wn\&id=912 (consultado en marzo de 2014).
Stavenhagen, Rodolfo. 2007. La cuestión étnica. México: COLMEX

Taylor, Charles. 1993. El multiculturalismo y la política del reconocimiento, México: FCE.

Toledo, Víctor. 1996. "Todas las Aguas. El subsuelo, las riberas, las tierras. Notas acerca de la (des)protección de los derechos indígenas sobre sus recursos naturales y contribución a una política pública de defensa". Disponible en: mapuche.info/ mapuint/aguas1.htm (consultado en febrero 2015). 
